\title{
Digital Libraries, Personalisation, and Network Effects - Unpicking the Paradoxes
}

\author{
Joy Palmer ${ }^{1}$, Caroline Williams ${ }^{1}$, Paul Walk ${ }^{2}$, David Kay ${ }^{3}$, Dean Rehberger ${ }^{4}$, \\ and Bill Hart-Davidson ${ }^{5}$ \\ ${ }^{1}$ JISC National Data Centre, UK \\ \{joy.palmer, caroline.williams\} @manchester.ac.uk \\ ${ }^{2}$ UKOLN, University of Bath, UK \\ p.walk@ukoln.ac.uk \\ ${ }^{3}$ Sero Consulting Ltd., UK \\ david.kay@sero.co.uk \\ ${ }^{4}$ Digital Humanities Research Centre, Michigan State University, USA \\ mfegan@mail.matrix.msu.edu \\ ${ }^{5}$ Writing in Digital Environments Research Center (WIDE), \\ Michigan State University, USA \\ hartdav2@msu . edu
}

\section{Panel Description}

The focus of this panel presentation is on personalisation (including adaptive personalisation) and the constructions of 'digital societies' around digital libraries and collections. Panelists will represent a variety of perspectives - NEH (USA) JISC (UK) \& EU - ranging from developers of highly specialised academic digital libraries, to directors of national digital libraries that aim to achieve system-wide aims.

The 'Library 2.0' and emerging 'Archives 2.0' movements are understandably asking what library and archives can do to exploit 2.0 and adaptive technologies to potentially enhance catalogues, finding aids and repositories. User-generated content, especially from subject experts, has the potential to enrich catalogue entries and digital objects and aid the processes of learning, teaching and research. Collections thus become potential 'architectures of participation'; the bases for robust digital communities that support education and research. In addition, questions are being asked over the role of attention data in personalisation, which can be used to widen participation and strengthen those communities, as well as providing new pathways through resources, yielding 'long tails' of digital library content. Webscale systems such as Google and Amazon can leverage network effects in astoundingly efficient ways, aggregating individual and group data to enhance search and adaptive personalisation functions. How might national digital libraries adapt such a model to support learning and research?

This panel will discuss the technical and cultural challenges that emerge when the Amazon-like model for supporting adaptive personalisation is transferred to the digital library domain. Technical challenges concern the significant shift in approaches to application development and 'system-wide' initiatives, approaches which increasingly privilege a dispersed and fragmented data-model. The drive for centralised digital library systems is being questioned, as a growing trend emerges whereby web technologies are used to knit or mash data and systems together at the presentation level. 